\title{
Charged-Hadron Pseudorapidity Distributions in p-p and Pb-Pb Collisions at LHC Energies
}

\author{
Bao-Chun Li and Ya-Zhou Wang \\ Department of Physics, Shanxi University, Taiyuan, Shanxi 030006, China \\ Correspondence should be addressed to Bao-Chun Li; libc2010@163.com
}

Received 26 June 2013; Revised 22 July 2013; Accepted 23 July 2013

Academic Editor: Fu-Hu Liu

Copyright ( 2013 B.-C. Li and Y.-Z. Wang. This is an open access article distributed under the Creative Commons Attribution License, which permits unrestricted use, distribution, and reproduction in any medium, provided the original work is properly cited.

Pseudorapidity distributions of charged hadrons produced in $\mathrm{p}-\mathrm{p}$ and $\mathrm{Pb}-\mathrm{Pb}$ collisions at $\mathrm{LHC}$ energies were measured by the CMS and ALICE Collaborations, respectively. An improved Tsallis distribution in the two-cylinder model is used to describe the pseudorapidity spectra. We consider the rapidity shift at the longitudinal direction in the geometrical picture of the collisions. The calculated results are in agreement with the experimental data. The gap between the projectile cylinder and the target cylinder increases with the centralities. The rapidity shifts in the cylinders also increase with the centralities.

\section{Introduction}

The Large Hadron Collider (LHC) at CERN has been built to research the properties of matter produced in high-energy collisions $[1,2]$. It will study proton-proton collisions at a center-of-mass energy of $14 \mathrm{TeV}$ and heavy-ion collisions at a center-of-mass energy of $5.5 \mathrm{TeV}$, which are much higher than the maximum collision energy at RHIC. An environment of high temperature and density is formed in such high-energy collisions, which lead to a significant extension of the kinematic range in longitudinal rapidity and transverse momentum [3-5]. The investigation of the particle production in proton-proton and nucleus-nucleus collisions at LHC energies is helpful for understanding the statistical behavior of particles and production mechanism. The multiplicity and pseudorapidity distributions of finalstate particles can be used to test different theoretical models and ideas. In the central rapidity region, the multiplicity of charged particles produced in the high-energy collisions is an important observable and can give the basic attribution of quark-gluon plasma (QGP) produced in the collisions. Proton-proton collisions at $7 \mathrm{TeV}$ produce about 70 charged hadrons integrated over the full rapidity space, including the unmeasured region. The study on the pseudorapidity distribution of charged hadrons, $d N_{(\mathrm{ch})} / d \eta$, helps us to understand the production mechanism of major charged hadrons.

The relativistic diffusion model (RDM) [6] has made some valuable attempts in describing and predicting pseudorapidity distribution of charged hadrons produced in heavyion collisions at SPS, RHIC, and LHC energies. Different phenomenological models of initial coherent multiple interactions and particle transport have been introduced to describe the production of final-state particles in $\mathrm{Au}-\mathrm{Au}$ collisions $[7,8]$. In the analysis of the experimental data, one statistical distribution gained prominence with very good fits to the data measured by the STAR [9] and PHENIX [10] Collaborations at RHIC and measured by the CMS [11] and ALICE [12] Collaborations at LHC. With Tsallis statistics' development and success in dealing with nonequilibrated complex systems in condensed matter research, it has been utilized to understand the particle production in highenergy collisions. Recently, in order to describe transverse momentum spectra, an improved Tsallis distribution which satisfies better the thermodynamic consistency was proposed [13]. As the collision energy increases to LHC, which is much higher than the maximal collision energy at RHIC, the kinematic range in the longitudinal direction will increase. In this work, we consider the rapidity shift of the interacting system and use the improved Tsallis distributions to analyze 
the pseudorapidity distribution functions in $\mathrm{p}-\mathrm{p}$ and $\mathrm{Pb}-\mathrm{Pb}$ collisions at LHC energies as measured by the CMS and ALICE Collaborations.

\section{The Improved Tsallis Distribution and the Rapidity Distribution}

In the framework of Tsallis statistic, more than one version of the Tsallis distribution is used to discuss the transverse momentum distribution of final-state particles produced in high-energy collisions. The improved form of the Tsallis distribution can naturally meet the thermodynamic consistency. The quantum form of the Tsallis distribution succeeded in description of the transverse distribution measured by ALICE and CMS Collaborations. According to the framework, the momentum distribution is given by

$$
E \frac{d^{3} N}{d^{3} P}=\frac{g V E}{(2 \pi)^{3}}\left[1+(q-1) \frac{E-\mu}{T}\right]^{-q /(q-1)},
$$

where $p, E, T, \mu, V$, and $g$ are the momentum, the energy, the temperature, the chemical potential, the volume, and the degeneracy factor, respectively, and $q$ is a parameter characterizing the degree of nonequilibrium. For zero chemical potential, a rapidity distribution is

$$
\begin{aligned}
\frac{d N}{d y}=g V \int & \frac{d P_{T}}{(2 \pi)^{2}} P_{T} m_{T} \cosh y \\
& \times\left[1+(q-1) \frac{m_{T} \cosh y}{T}\right]^{-q /(q-1)},
\end{aligned}
$$

where $P_{T}$ is the transverse momentum. The distribution function is only the rapidity distribution of particles emitted in a considered emission source at the fixed rapidity. In $y$-space, the longitudinal location of the source needs to be taken into account. Therefore, for the fixed emission source with rapidity $y_{x}$, the rapidity distribution of produced particles is given by

$$
\begin{aligned}
& f\left(y, y_{x}\right)= \frac{d N}{d y} \\
&=g V \int \frac{d P_{T}}{(2 \pi)^{2}} P_{T} m_{T} \cosh \left(y-y_{x}\right) \\
& \\
& \quad \times\left[1+(q-1) \frac{m_{T} \cosh \left(y-y_{x}\right)}{T}\right]^{-q /(q-1)} .
\end{aligned}
$$

Generally speaking, the parameters $T$ and $q$ are obtained by fitting the transverse spectra measured in the collisions. In [13], the values of $T$ and $q$ taken for the calculations are about $0.07 \mathrm{GeV}$ and 1.1 , respectively.

In order to describe the rapidity shift, we introduce the geometrical picture of the two-cylinder model [16]. In the laboratory reference system, the projectile cylinder is in the positive rapidity direction and the target cylinder is in the negative one, with rapidity ranges $\left[y_{p \min }, y_{p \max }\right]$ and $\left[y_{t \min }, y_{t \max }\right]$, respectively. On both sides of the two cylinders there are leading particles appearing as two isotropic emission sources with rapidity shifts $y_{P}$ and $y_{T}$, respectively. So, in the final state, the normalized pseudorapidity distribution is

$$
\begin{aligned}
f(y)= & k_{t} f\left(y, y_{T}\right)+\frac{K_{t}}{y_{t \max }-y_{t \min }} \\
& \times \int_{y_{t \min }}^{y_{t \max }} f\left(y, y_{t}\right) d y_{t}+\frac{K_{p}}{y_{p \max }-y_{p \min }} \\
& \times \int_{y_{p \min }}^{y_{p \max }} f\left(y, y_{p}\right) d y_{p}+k_{p} f\left(y, y_{P}\right),
\end{aligned}
$$

where $k_{t}, K_{t}, K_{p}$, and $k_{p}$ are the contributions of the target leading particles, the target cylinder, the projectile cylinder, and the projectile leading particles, respectively. For symmetric collisions $\mathrm{p}-\mathrm{p}$ and $\mathrm{Pb}-\mathrm{Pb}, K_{t}=K_{p}=k, k_{t}=k_{p}=$ $1-k$. The pseudorapidity distribution can be expressed as

$$
\begin{aligned}
f(y)= & \frac{k}{y_{p \max }-y_{p \min }} \\
& \times\left[\int_{y_{p \min }}^{y_{p \max }} f\left(y, y_{p}\right) d y_{p}+\int_{-y_{p \max }}^{-y_{p \min }} f\left(y, y_{t}\right) d y_{t}\right] \\
& +\frac{(1-k)}{2}\left[f\left(y, y_{P}\right)+f\left(y,-y_{P}\right)\right] .
\end{aligned}
$$

The pseudorapidity distribution can be calculated by a conversion between the pseudorapidity distribution and the rapidity distribution. In the case of $p \gg m_{0}$, the rapidity $y$ and pseudorapidity $\eta$ are approximately equal to each other. However, the condition of $p \gg m_{0}$ is not always satisfied. The conversion between the pseudorapidity distribution $d N / d \eta$ and the rapidity distribution $d N / d y$ is

$$
\frac{d N}{d \eta}=\frac{p}{E} \frac{d N}{d y}=J\left(\eta, \frac{\langle m\rangle}{\left\langle p_{T}\right\rangle}\right) \frac{d N}{d y},
$$

where a Jacobian of the transformation is

$$
J\left(\eta, \frac{\langle m\rangle}{\left\langle p_{T}\right\rangle}\right)=\frac{\cosh \eta}{\sqrt{1+\left(\langle m\rangle /\left\langle p_{T}\right\rangle\right)^{2}+\sinh ^{2} \eta}} .
$$

\section{Comparison with Experimental Results}

Figure 1 presents the pseudorapidity distributions of charged particles produced in $\mathrm{p}$-p collisions at $\sqrt{\boldsymbol{s}_{N N}}=2.36 \mathrm{TeV}$ and $7 \mathrm{TeV}$. The symbols represent the experimental data of the CMS Collaboration [5] and the curves are our calculated results. The parameters used in the calculations are taken to be $y_{p \max }=4.562 \pm 0.140, y_{p \min }=0.224 \pm 0.009$ and $y_{P}=4.480 \pm 0.127$ and $y_{p \max }=5.146 \pm 0.170$, and $y_{\text {pmin }}=0.254 \pm 0.011$ and $y_{P}=4.760 \pm 0.159$, respectively. The parameter $k$ is taken at the same value $0.432 \pm 0.012$. The $\chi^{2}$ per degree of freedom (dof) are 0.454 and 0.612 , respectively. The calculated results are in good agreement 


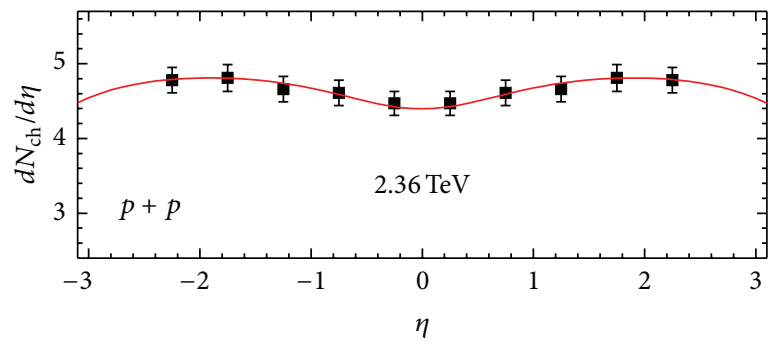

(a)

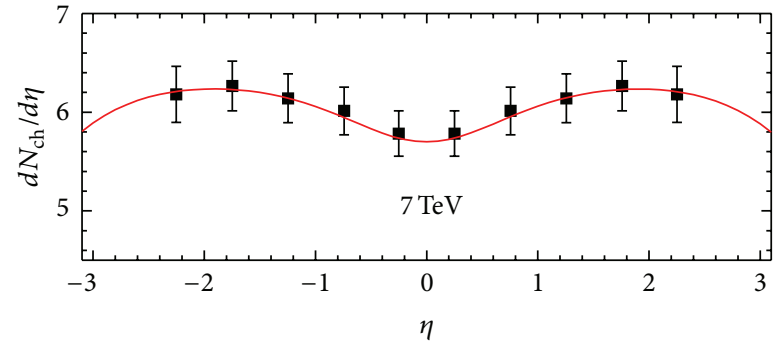

(b)

Figure 1: (Color online) The charged particle multiplicity $d N_{\mathrm{ch}} / d \eta$ in p-p inelastic collisions at $\sqrt{s_{N N}}=2.36 \mathrm{TeV}$ and $7 \mathrm{TeV}$. The symbols represent the experimental data measured by the CMS Collaboration [5]. The curves are our calculated results.

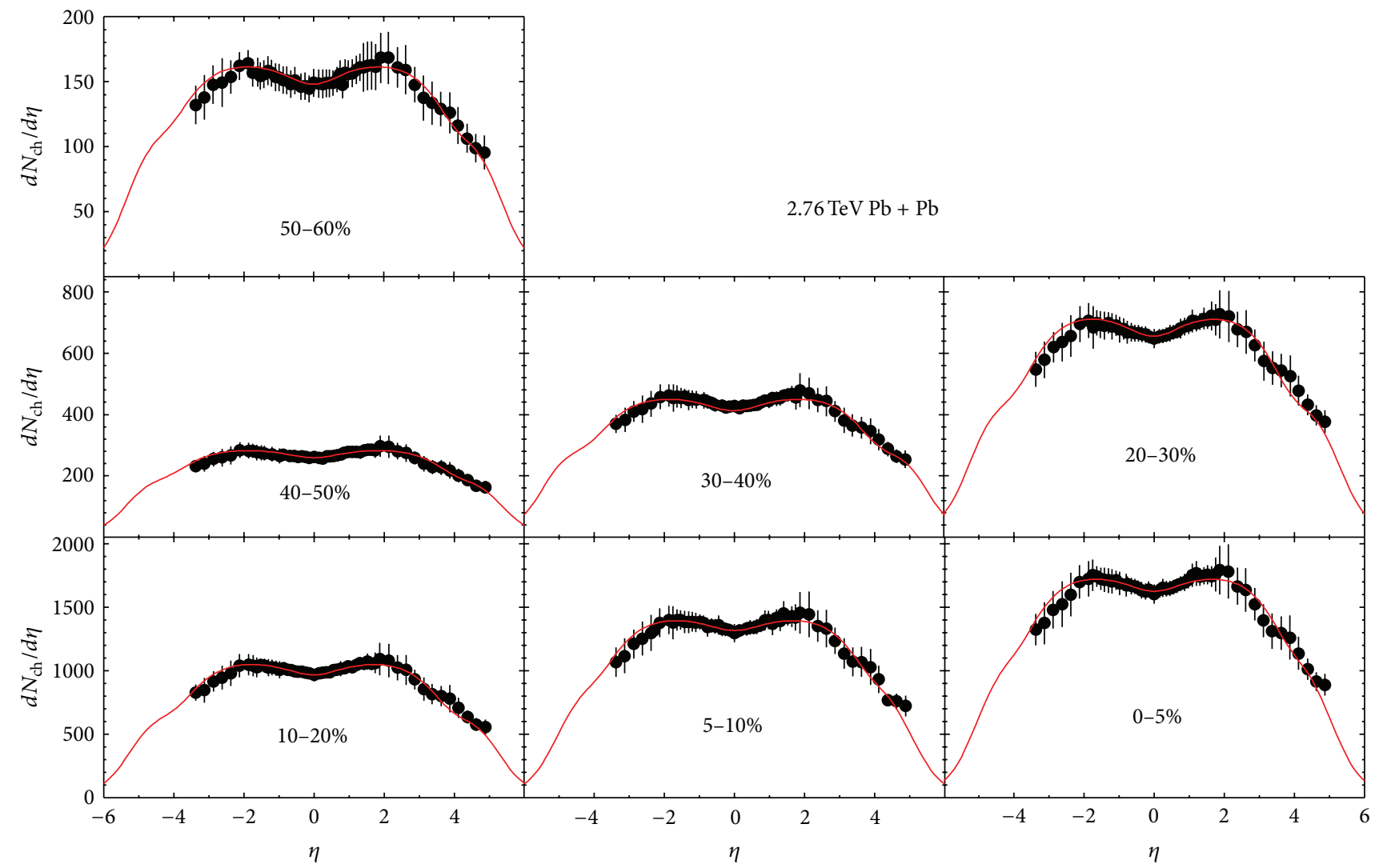

Figure 2: (Color online) The charged particle multiplicity $d N_{\mathrm{ch}} / d \eta$ for different centrality bins in $\mathrm{Pb}-\mathrm{Pb}$ collisions at $\sqrt{s_{N N}}=2.76 \mathrm{TeV}$. Experimental data of the ALICE Collaboration $[14,15]$ are shown by the scattered symbols. Our calculated results are shown by the curves.

with the experimental data. The rapidity shifts $y_{p \max }-y_{p \min }$ in the cylinders for $7 \mathrm{TeV}$ are larger than that for $2.36 \mathrm{TeV}$. So is the gap between the projectile cylinder and the target cylinder $2 y_{\text {pmin }}$.

Figure 2 shows the pseudorapidity distributions of charged particles produced in $\mathrm{Pb}-\mathrm{Pb}$ collisions with different centralities at $\sqrt{s_{N N}}=2.76 \mathrm{TeV}$. The values of centralities are shown in the figure. The symbols represent the experimental data of the ALICE Collaboration $[14,15]$ and the curves are our calculated results. The value of $k$ is $0.421 \pm 0.010$.
The other parameters $y_{p \max }, y_{p \min }, y_{P}$, and $N_{c}$ obtained by fitting the experimental data are given in Table 1 with $\chi^{2} /$ dof. From these values, we find that the $y_{p \max }$ and $y_{p \min }$ increase with the increase in the centralities. In other words, the length of the double cylinder and the distance between the two cylinders increase with the increase in the centralities. The maximum value of $\chi^{2} /$ dof is 1.156 . One sees that the calculated results approximately agree with the experimental data for all concerned centralities. The dependences of the different parameters on the centrality class are given in Figure 3. 

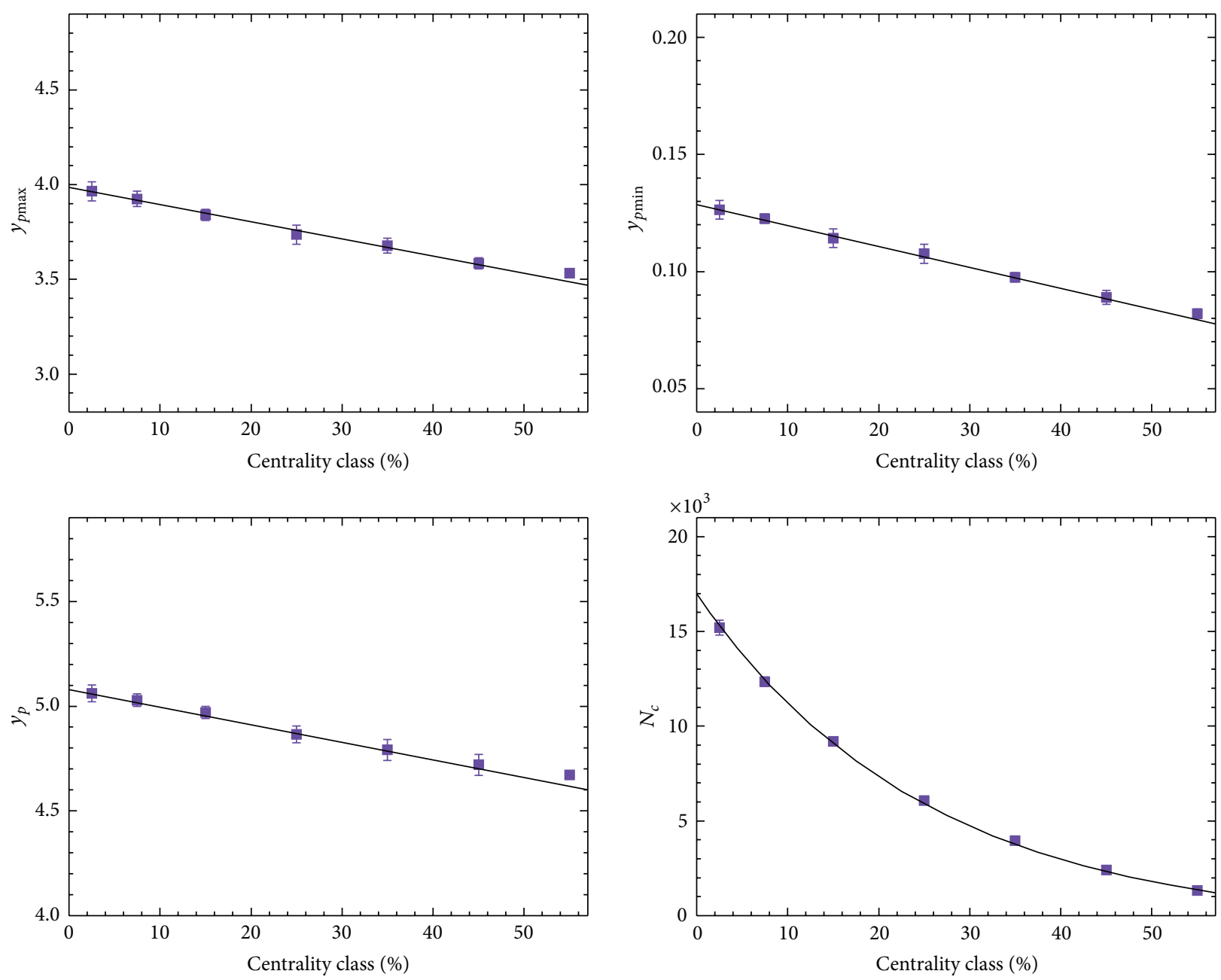

FIgure 3: (Color online) The dependence of the different parameters on the centrality class. The symbols represent the parameter values used in Figure 2. The solid lines are fitted results.

TABLE 1: Parameter values corresponding to the solid curves in Figure 2.

\begin{tabular}{|c|c|c|c|c|c|c|}
\hline Centrality & $y_{p \max }$ & $y_{p \min }$ & $y_{P}$ & $k$ & $N_{c}$ & $\chi^{2} / \mathrm{dof}$ \\
\hline $0-5 \%$ & $3.965 \pm 0.050$ & $0.126 \pm 0.004$ & $5.060 \pm 0.040$ & $0.420 \pm 0.050$ & $15200 \pm 400$ & 0.925 \\
\hline $5-10 \%$ & $3.925 \pm 0.040$ & $0.123 \pm 0.002$ & $5.028 \pm 0.030$ & $0.420 \pm 0.050$ & $12350 \pm 240$ & 0.805 \\
\hline $10-20 \%$ & $3.841 \pm 0.030$ & $0.114 \pm 0.004$ & $4.971 \pm 0.030$ & $0.420 \pm 0.050$ & $9200 \pm 180$ & 0.778 \\
\hline $20-30 \%$ & $3.736 \pm 0.050$ & $0.108 \pm 0.004$ & $4.865 \pm 0.040$ & $0.420 \pm 0.050$ & $6080 \pm 140$ & 1.156 \\
\hline $30-40 \%$ & $3.678 \pm 0.040$ & $0.098 \pm 0.002$ & $4.791 \pm 0.050$ & $0.420 \pm 0.050$ & $3950 \pm 80$ & 0.718 \\
\hline $40-50 \%$ & $3.585 \pm 0.030$ & $0.089 \pm 0.003$ & $4.721 \pm 0.050$ & $0.420 \pm 0.050$ & $2405 \pm 68$ & 0.627 \\
\hline $50-60 \%$ & $3.533 \pm 0.020$ & $0.082 \pm 0.002$ & $4.671 \pm 0.020$ & $0.420 \pm 0.050$ & $1340 \pm 46$ & 0.644 \\
\hline
\end{tabular}

By fitting the data, the function relations between the different parameters and the centrality class are determined:

$$
\begin{gathered}
y_{p \max }=-0.902 c+3.984, \\
y_{p \min }=-0.089 c+0.129, \\
y_{P}=-0.841 c+5.079, \\
N_{c}=17580.5 e^{-c / 0.251}-600.4,
\end{gathered}
$$

where $c$ and $N_{c}$ denote the centrality and a normalization constant, respectively. The values of $\chi^{2} /$ dof are $0.819,0.652$, 0.736 , and 0.441 , respectively.

\section{Discussions and Conclusions}

In the above comparisons, we have investigated the pseudorapidity distributions of charged hadrons produced in $\mathrm{p}-\mathrm{p}$ and $\mathrm{Pb}-\mathrm{Pb}$ collisions at LHC energies. In [13], the improved Tsallis 
distribution which satisfies the thermodynamic consistency was proposed to fit the experimental data. However, it can only treat the transverse momentum spectra, but not the rapidity (or pseudorapidity) distributions. As the collision energy increases to LHC, which is much higher than the maximal collision energy at RHIC, the kinematic range in the longitudinal direction increases obviously. For the pseudorapidity distributions of charged hadrons, the rapidity shifts of emission sources in the interaction system have to be taken into account, which requires consistently the geometrical picture of the collisions. It is not difficult for the two-cylinder model to describe particle production in the rapidity space. We implemented the improved Tsallis distributions in the two-cylinder model and applied it to description of the pseudorapidity distributions. The calculated results are compared with the experimental data from the CMS and ALICE Collaborations. The calculated results are in agreement with the data, and the parameter dependence on the centrality is obtained. The gap between the projectile cylinder and the target cylinder increases with the centralities. The rapidity shifts in the cylinders also increase with the centralities.

The two-cylinder model was developed from the fireball model, which is suggested in heavy-ion collisions [17]. At the longitudinal position, the projectile cylinder and target cylinder are assumed to be formed in nucleus-nucleus (or proton-proton) collisions. At intermediate energy, the two cylinders overlap totally, and the interacting system is in fact described by a single cylinder model. At high energy, the two cylinders overlap partly. At ultrahigh energy, there is a gap appearing between the two cylinders. In the rapidity space, the projectile cylinder and target cylinder are defined to lie in the rapidity ranges $\left[y_{p \min }, y_{p_{\max }}\right]$ and $\left[y_{t \min }, y_{t \max }\right]$, respectively. It is expected that a thick double cylinder is formed in nucleus-nucleus collisions and a thin double cylinder is formed in nucleon-nucleon collisions. The collision picture is very intuitive and accessible.

In conclusion, the experimental pseudorapidity distributions of charged hadrons produced in $\mathrm{p}-\mathrm{p}$ and $\mathrm{Pb}-\mathrm{Pb}$ collisions at LHC energies have been described by the improved Tsallis distributions in the two-cylinder model. The calculated results show that the rapidity shifts $y_{p \max }$ and $y_{p \min }$ increase with the increase in the centralities, whereas the contributions of the projectile and target cylinders to the pseudorapidity distributions do not change obviously with the increase in the centralities. The length of the projectile cylinder or the target cylinder increases and the gap between them increases with the increase in the centralities.

\section{Acknowledgments}

This work is supported by the National Natural Science Foundation of China under Grant nos. 11247250, 11005071, and 10975095; the National Fundamental Fund of Personnel Training under Grant no. J1103210; the Shanxi Provincial Natural Science Foundation under Grants nos. 2013021006 and 2011011001; the Open Research Subject of the Chinese Academy of Sciences Large-Scale Scientific Facility under
Grant no. 2060205; and the Shanxi Scholarship Council of China.

\section{References}

[1] S. Chatrchyan, V. Khachatryan, A. M. Sirunyan et al., "Indications of suppression of excited $Y$ states in $\mathrm{Pb}-\mathrm{Pb}$ collisions at $\sqrt{s_{N N}=2.76}$ TeV," Physical Review Letters, vol. 107, no. 5, Article ID 052302, 15 pages, 2011.

[2] S. Chatrchyan, V. Khachatryan, A. M. Sirunyan et al., "Study of $\mathrm{Z}$ boson production in $\mathrm{PbPb}$ collisions at nucleon-nucleon centre of mass energy $=2.76 \mathrm{TeV}$," Physical Review Letters, vol. 106, Article ID 212301, 22 pages, 2011.

[3] R. Aaij, C. A. Beteta, A. Adametz et al., "Measurement of prompt hadron production ratios in $p p$ collisions at $\sqrt{s=0.9}$ and $7 \mathrm{TeV}$," European Physical Journal C, vol. 72, article 2168, 2012.

[4] K. Aamodt, N. Abel, U. Abeysekara et al., "Transverse momentum spectra of charged particles in proton-proton collisions at $\sqrt{s=900} \mathrm{GeV}$ with ALICE at the LHC," Physics Letters B, vol. 693, pp. 53-86, 2010.

[5] V. Khachatryan, A. M. Sirunyan, A. Tumasyan et al., "Transverse-momentum and pseudorapidity distributions of charged hadrons in $p p$ collisions at $\sqrt{s=7} \mathrm{TeV}$," Physical Review Letters, vol. 105, no. 2, Article ID 022002, 14 pages, 2010.

[6] G. Wolschin, "Charged-hadron pseudorapidity distributions in the RDM," in Proceedings of the 7th Workshop on Particle Correlations and Femtoscopy, vol. 061, Tokyo, Japan, September 2011.

[7] P. Braun-Munzinger, D. Magestro, K. Redlich, and J. Stachel, "Hadron production in Au-Au collisions at RHIC", Physics Letters B, vol. 518, no. 1-2, pp. 41-46, 2001.

[8] J. Rafelski and J. Letessier, "Testing limits of statistical hadronization," Nuclear Physics A, vol. 715, pp. 98c-107c, 2003.

[9] B. I. Abelev, J. Adams, M. M. Aggarwal et al., "Strange particle production in $p+p$ collisions at $\sqrt{s_{N N}=200} \mathrm{GeV}$," Physical Review Letters C, vol. 75, Article ID 064901, 2007.

[10] A. Adare, S. Afanasiev, C. Aidala et al., "Identified charged hadron production in $p+p$ collisions at $\sqrt{s=200}$ and 6204 GeV,' Physical Review Letters C, vol. 83, Article ID 064903, 32 pages, 2011.

[11] V. Khachatryan, A. M. Sirunyan, A. Tumasyan et al., "Strange particle production in pp collisions at $\sqrt{s=0.9}$ and $7 \mathrm{TeV}$," Journal of High Energy Physics, vol. 1105, article 064, 2011.

[12] K. Aamodt, N. Abel, U. Abeysekara et al., "Production of pions, kaons and protons in pp collisions at $\sqrt{s=900} \mathrm{GeV}$ with ALICE at the LHC," European Physical Journal C, vol. 71, article 1655, 30 pages, 2011.

[13] J. Cleymans and D. Worku, "Relativistic thermodynamics: transverse momentum distributions in high-energy physics," European Physical Journal A, vol. 48, article 160, 2012.

[14] A. Toia, "Bulk properties of $\mathrm{Pb}-\mathrm{Pb}$ collisions at $\sqrt{s_{N N}=2.76} \mathrm{TeV}$ measured by ALICE," Journal of Physics G, vol. 38, no. 12, Article ID 124007, 2011.

[15] D. Roehrscheid and G. Wolschin, "Centrality dependence of charged-hadron pseudorapidity distributions in $\mathrm{PbPb}$ collisions at LHC energies in the RDM," Physical Review Letters C, vol. 86, Article ID 024902, 8 pages, 2012.

[16] F. H. Liu, "Particle production in Au-Au collisions at RHIC energies," Physics Letters B, vol. 583, no. 1-2, pp. 68-72, 2004. 
[17] G. D. Westfall, J. Gosset, P. J. Johansen et al., "Nuclear fireball model for proton inclusive spectra from relativistic heavy-ion collisions," Physical Review Letters, vol. 37, no. 18, pp. 1202-1205, 1976. 

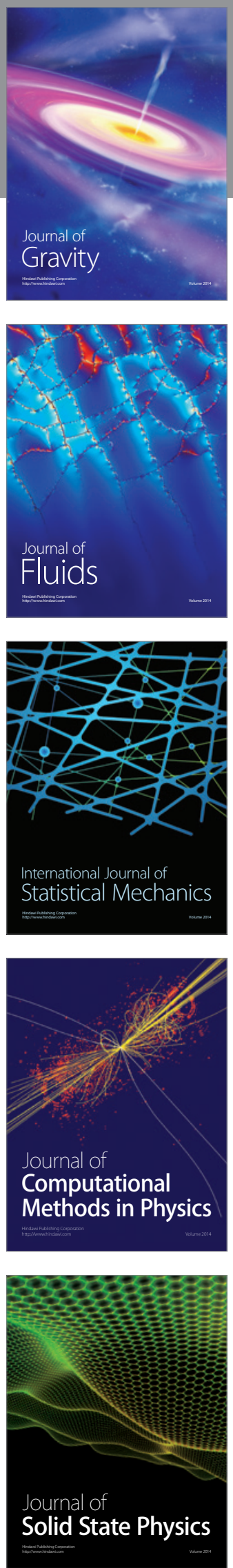

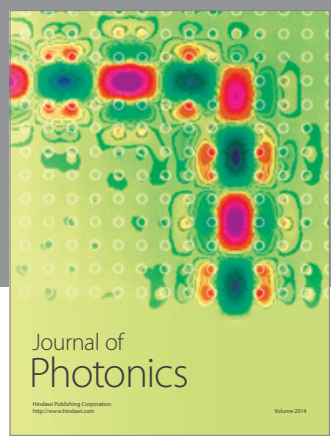

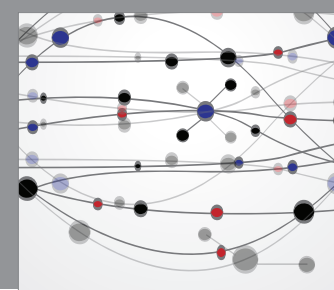

The Scientific World Journal

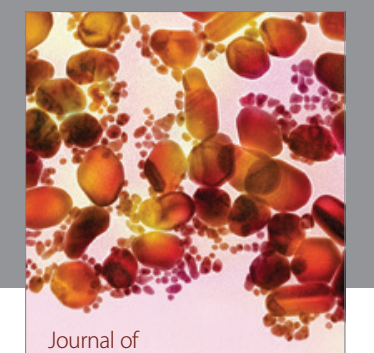

Soft Matter
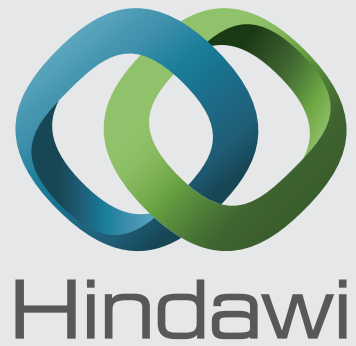

Submit your manuscripts at

http://www.hindawi.com
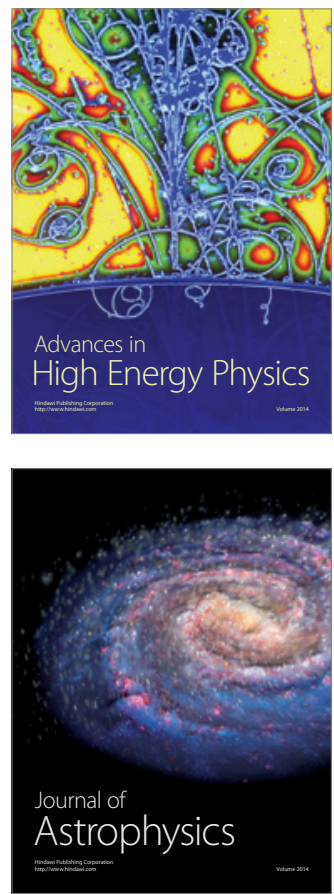
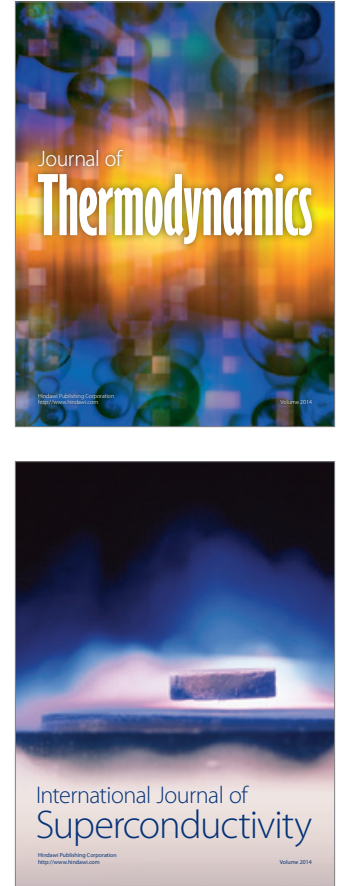
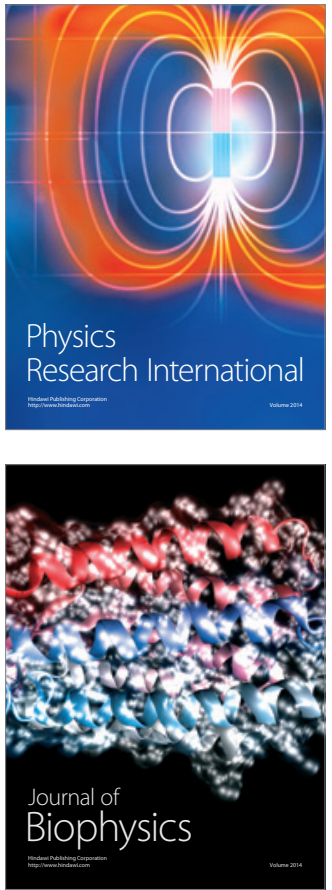
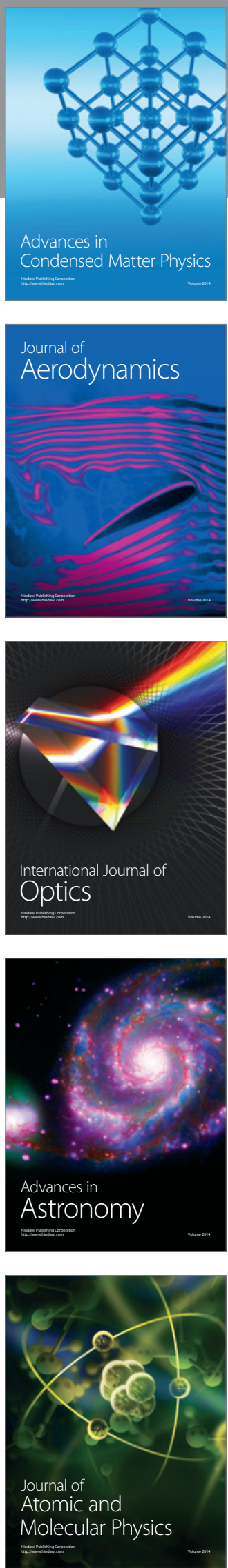